\title{
Le point de vue du médecin du travail
}

\author{
J. BINDER, E. DOSSIER, J.-L. MARANDE
}

Selon la réglementation française (voir chapitre 4), les résultats de dosimétrie passive sont considérés comme éléments du dossier médical de chaque travailleur exposé et sont donc destinés exclusivement au médecin du travail qui doit les communiquer aux travailleurs à leur demande et, de façon systématique, lors de la visite médicale annuelle. La notion de confidentialité de ces résultats est spécifiquement française et parfois vivement contestée. Cependant, lors des longues discussions avec les partenaires sociaux pour rédiger le décret du 31 mars 2003, ce principe n'a pas été remis en cause.

Du point de vue pratique, jusqu'à l'avènement de SISERI, cette disposition n'était pas facile à appliquer pour les laboratoires de dosimétrie généraliste qui, ne pouvant disposer d'un fichier stable des médecins du travail, adressaient les résultats à l'adresse de l'établissement sous pli confidentiel cacheté, à l'attention du médecin du travail. Ces résultats avaient parfois du mal à parvenir à leurs destinataires réglementaires.

Sur les différents aspects de ce suivi dosimétrique les docteurs Jean Binder, Ellen Dossier et Jean-Luc Marande ont accepté de faire part de leurs expériences.

\subsection{L'expérience du docteur Jean Binder, médecin du travail à Framatome puis à AREVA}

La surveillance dosimétrique des salariés de Framatome, depuis 1975, dès la création de l'usine de Saint-Marcel, différenciait la dosimétrie passive de la dosimétrie active ou opérationnelle. La dosimétrie passive était assurée par le dosifilm ou film dosimétrique et la dosimétrie active était assurée par des stylosdosimètres.

La population surveillée était constituée au départ des contrôleurs radio mettant en œuvre des générateurs de rayons $X$, un accélérateur linéaire de rayons $X$ de 6 , 9 et $11 \mathrm{MeV}$ et la gammagraphie industrielle à l'aide de sources scellées de cobalt 60 et d'iridium 192. 
Avec le développement du programme nucléaire français des réacteurs PWR, réacteurs de 900, 1300, 1450 mégawatts, la maintenance nucléaire s'est développée de façon exponentielle au point de devenir très vite une activité séparée de l'usine de fabrication des composants primaires des centrales nucléaires.

En centrale nucléaire, les radioexpositions étaient les premières années essentiellement liées aux produits d'activation, émetteurs bêta-gamma. Pendant très longtemps le film dosimétrique a été la méthode de dosimétrie passive mise en œuvre en faisant appel au laboratoire de dosimétrie du SCPRI, puis de l'OPRI qui en était, en 1994, la continuité. Le premier constructeur mondial de centrales nucléaires se devait d'être abonné au SCPRI et nous nous souvenons d'avoir signé vers la fin des années 1970 le Livre d'or de cette institution, inaugurant ainsi ses relations avec le monde de l'industrie. Nous nous souvenons aussi d'un responsable du service radioprotection de Framatome appelé, alors qu'il était en mission aux États-Unis, par son PDG pour lui demander de renoncer immédiatement à la résiliation de son contrat avec le SCPRI... Ce fut de toute évidence une initiative malheureuse. Un simple appel téléphonique du Professeur Pierre Pellerin avait suffit.

En dosimétrie opérationnelle, les premiers dosimètres électroniques ont supplanté les stylos-dosimètres, du moins en INB, alors qu'il faudra bien plus de temps pour y arriver en usine. Le film avait pour nous médecin un certain nombre d'avantages : possibilité de relecture, information sur le type d'exposition, l'énergie des rayonnements, information sur une exposition statique liée à son oubli ou sa perte en zone contrôlée, irradiation lors d'un contrôle en douane ou lors de tri postal, ce qui se vit au départ en périodes d'attentats, puis ces dernières années de manière plus systématique dans les aéroports. Nous avons ainsi découvert qu'il existait une grande hétérogénéité des appareils utilisés et qu'il pouvait y avoir aussi des contrôles approfondis plus irradiants... Ses marges d'imprécision nous étaient connues. On savait qu'à 50 mrem $(0,50 \mathrm{mSv})$, on parlait en effet en rem et en millirem à cette époque, on avait $100 \%$ d'incertitude, qu'à 5 rems $(50 \mathrm{mSv})$ on avait 10 à $15 \%$ d'incertitude, et qu'à 400 mrem $(4 \mathrm{mSv})$, on avait la meilleure précision : $4 \%$ d'incertitude. Et la Publication 35 de la CIPR nous rassurait on ne peut plus en considérant que l'on faisait une dosimétrie de bonne qualité si les résultats pour l'intervalle de confiance $95 \%$ étaient dans la plage $-33+50 \%$ autour de 5 rems $(0,05 \mathrm{~Sv})$ et $\quad-50+100 \%$ autour de $1 \mathrm{rem}(0,01 \mathrm{~Sv})$. Il n'en demeurait pas moins que les imprécisions de lecture pouvaient être considérablement réduites et le laboratoire de dosimétrie du SCPRI dirigé par Alain Biau, avec qui nous avions de très fréquents contacts, nous en donnera longtemps la preuve.

En novembre 1982, Framatome a mis en place une dosimétrie passive parallèle avec des dosimètres radiothermoluminescents (pastilles ${ }^{7} \mathrm{LiF}$ ) permettant 
d'anticiper la connaissance de la dose mensuelle vu la lenteur des délais de restitution de la lecture des films. Cette dosimétrie par pastilles de LiF, lues en interne, servait également pour des dosimétries complémentaires, notamment dose poignet ou dose extrémité par bagues LiF. La précision de cette dosimétrie radiothermoluminescente s'est avérée extrêmement intéressante, de quelques pour cent, 3 à $4 \%$, au-delà de la connaissance anticipée des doses mensuelles. Cela a permis d'affiner la précision de la dosimétrie film en demandant des relectures pour tous les résultats films ayant des écarts significatifs. Notre connaissance de la dosimétrie opérationnelle jouait de plus en notre faveur. Cela fit les beaux jours du responsable du laboratoire de dosimétrie du SCPRI puis de l'OPRI. Il y eut des erreurs de lecture liées à des raisons ou problèmes techniques, dans un sens comme dans l'autre, même si la dosimétrie radiothermoluminescente était souvent la plus exacte. Cela nous permit aussi d'avoir la chance d'un traitement de faveur, car connaissant nos moyens, le SCPRI décida de relire tous les dosifilms venant de Framatome qui présentaient une dose significative. Du photodensitomètre à l'œil humain... Malgré une méthodologie indéniablement remarquable, ces échanges sur de longues années ont permis d'assurer un haut niveau de suivi dosimétrique pour l'une des populations de travailleurs les plus exposés de France. Et cela permit aussi de déceler des dysfonctionnements liés au vieillissement du matériel de l'organisme de dosimétrie.

Très tôt également, dès le début des années 1980, une restitution mensuelle à chaque intervenant de ses résultats dosimétriques, sous couvert du service médical, fut mise en place à Framatome par gestion informatique : résultats du mois en cours ou des deux derniers mois en dose LiF, cumul 12 mois, cumul 5 ans et cumul vie avec la prise en compte aussi des seules dosimétries film.

Cela équivalait à la fiche d'exposition (dosimétrique) devenue fiche de résultats dosimétriques par une curieuse évolution sémantique, alors que la fiche d'exposition est devenue une fiche récapitulant les expositions à des nuisances ou risques professionnels. La gestion de l'emploi par la dose n'étant pas un problème, du fait du type de contrat de travail, il n'y avait pas d'états d'âme à avoir pour procéder ainsi. Et à ceux qui militaient pour considérer que la dosimétrie passive relevait du secret médical, il suffisait de rappeler qu'en cas de dépassement de dose, il fallait informer l'employeur, le Comité d'hygiène, de sécurité et des conditions de travail (CHSCT), l'Inspection du travail... Ce qui constituait de fait une levée du secret... Cacher des doses nulles ou très faibles et tout révéler si on est en infraction avec la loi, donc trop tard, on ne peut pas dire que cela allait dans le sens de la radioprotection, même si l'optimisation et la limitation des doses se faisaient déjà sur la dosimétrie opérationnelle. 
Le temps passa. Framatome devint AREVA le 3 septembre 2001. L'OPRI avait pour sa part cédé sa place à l'IRSN en février 2002. Le dosifilm était condamné et sa pertinence remise en cause au vu des nouveaux types de dosimètres qui allaient être mis sur le marché. Les services nucléaires d'AREVA optèrent comme EDF pour la technologie OSL, dosimètre InLight, du LCIE Landauer en janvier 2005. Le retard pris par l'IRSN dans le choix et la mise en service d'une dosimétrie par technique radiophotoluminescente en est l'une des raisons. Des problèmes techniques, avec notamment du matériel déficient, trop longs à surmonter, depuis l'année 2002, véritable « année horrible », en était une autre. Deux ans plus tôt, en 2000 , on avait été confronté au vieillissement des dosimètres électroniques du parc électronucléaire français, avec des sous-estimations systématiques de doses, ce qui justifia leur remplacement par du matériel de nouvelle génération. À chacun sa croix... Et à chaque fois, la dosimétrie radiothermoluminescente, mise en place à Framatome à titre complémentaire, permettait de s'en apercevoir. Il nous est difficile encore pour le moment de discourir sur une meilleure réponse angulaire du dosimètre radiophotoluminescent. L'OSL nous semble convenir. Le dispositif Imaging nous a déjà permis de confirmer des expositions statiques par contrôles du courrier en douanes... Nous maintenons toujours la dosimétrie parallèle par dosimètres radiothermoluminescents. Les relectures que nous demandons chaque mois sont peu nombreuses et le plus souvent pour des doses très faibles.

Les doses reçues ont en effet considérablement baissées ces dix dernières années et nous en sommes à gérer réellement de très faibles doses. Indéniablement, dans cette évolution, il fallait passer à des techniques de dosimétrie plus précises dans les très faibles doses. Et l'objection selon laquelle le dosifilm présentait une surface d'exposition aux rayonnements ionisants beaucoup plus grande que les nouveaux détecteurs ne tient plus au regard de cela. L'évolution des radio-expositions dans les années 1990 nous a également confronté au risque neutrons. En dosimétrie passive, nous sommes passés en 35 ans du film «bêta gamma neutrons lents » du SCPRI, + neutrons rapides si nécessaire, au film «bêta gamma neutrons lents » associé à un détecteur solide de traces pour les neutrons rapides début 2000, puis en 2006 au Neutrak 144-T du LCIE Landauer qui couvre en principe le champ des neutrons rencontrés en centrales nucléaires. Reste là encore le problème de la réponse angulaire, même si par provocation nous disons qu'il faut toujours aborder les neutrons de face... Et nous n'avons pas suffisamment de justifications pour mettre en place une dosimétrie neutrons par radiothermoluminescence.

Parallèlement, nous sommes passés, en dosimétrie opérationnelle neutrons, des dosimètres à bulles aux dosimètres électroniques neutrons. Il n'en reste pas moins qu'il faut aujourd'hui comme hier gérer les pertes ou oublis de dosimètres passifs, remplacer les doses manquantes par la dosimétrie opérationnelle, veiller à ce que les dosimètres soient bien portés sur la période du mois civil, ni un peu avant, ni 
un peu au-delà, sinon cela fausse les intercomparaisons, gérer les prêts de dosimètres non nominatifs en les rendant nominatifs lors de leur envoi au laboratoire de dosimétrie, car un volant de dosimètres non nominatifs reste une nécessité dans le domaine des activités industrielles...

L'instauration en 1992 d'une carte professionnelle de suivi médical des travailleurs exposés aux rayonnements ionisants, initialement prévue par l'arrêté du 31 juillet 1991, délivrée par le médecin du travail, et ses évolutions successives, a constitué une première initiative d'identification nationale des travailleurs du nucléaire. La nécessité d'un numéro unique, conservé toute la vie professionnelle, s'est imposée par la suite, mais cela reste perfectible et un lien avec le suivi dosimétrique serait certainement utile.

La mise en place du système d'information de la surveillance de l'exposition aux rayonnements ionisants, avec la base de données dosimétriques SISERI en juillet 2005, alors que l'on y travaillait depuis 2002, recueille tout notre intérêt. L'intégration des dosimétries passées est plutôt fiable pour les salariés des grandes entreprises du nucléaire, encore que les résultats des dosimètres non nominatifs utilisés occasionnellement ne sont pour la plupart pas intégrés. Mais les fiches de résultats dosimétriques des dossiers médicaux les ont pris en compte et une confrontation des données est possible si cela est nécessaire.

Pour les PME-TPE du nucléaire, c'est souvent plus complexe et, à une époque ancienne, on a même vu des dosimètres nominatifs d'un salarié remis à un autre salarié sans que le laboratoire de lecture en ait été informé, sans compter de nombreux dosimètres non rendus sans recherche de la dosimétrie opérationnelle et sans enquête de la personne compétente en radioprotection. C'est une situation à considérer lorsque l'on initie une étude épidémiologique rétrospective ou une enquête cas-témoins, sachant que les doses seront dans ces situations toujours sousestimées.

Sur un plan prospectif, à long terme, SISERI présente un réel intérêt. Nous avons la preuve que cela fonctionne plutôt bien à présent, dès lors que les laboratoires qui transmettent les données le font de manière parfaite. Cela a mis un peu de temps, mais on y est arrivé. Le risque actuel, c'est que de plus en plus de médecins du travail, profession en baisse constante d'effectifs et totalement débordée sur le plan professionnel, se contentent d'éditer le cumul SISERI lors des visites médicales sans esprit critique, sans chercher à combler les pertes de dosimétrie passive, sans faire d'intercomparaisons. Un certain nombre de ces médecins considèrent en effet que le système national mis en place pour la dosimétrie passive les exonère de leurs responsabilités. Pas tout à fait vrai ou pas tout à fait faux ? Il y a une faille à ce niveau. 
Et pourtant, au-delà de tout ce que nous avons évoqué, la dosimétrie des rayonnements ionisants est un exemple unique de suivi dans le temps, de traçabilité d'une exposition professionnelle. On en est loin encore pour tous les autres risques physiques et les risques chimiques.

\subsection{L'expérience du docteur Ellen Dossier, médecin du travail du CNRS de 1974 à 2005}

Les médecins du travail dans la recherche scientifique ont très souvent travaillé en binôme avec les radioprotectionnistes et ont ainsi pu partager les résultats dosimétriques en temps utile dans un but de prévention accrue. Cela n'était pas l'esprit du législateur qui voulait insister sur le secret médical et la protection des salariés à l'embauche (dans les entreprises intérimaires de l'industrie notamment).

Dans la recherche, tout est artisanal, les licenciements n'existent pas, par contre il est prudent de ne pas exposer les équipes à des risques imprévus. C'est ainsi que nous avons longtemps affiché les résultats dosimétriques, toujours de faibles doses, avec l'accord des CHS, afin d'obtenir une rapide réactivité des manipulateurs, titulaires ou non, et collaborateurs de tous pays intervenants.

Les premiers médecins du travail, dès les années 1960, ont été très aidés par les enquêtes dosimétriques sur fantômes «truffés » de dosimètres lors des accidents d'exposition, lorsque des chercheurs s'étaient trouvés exposés brutalement en zone interdite, du fait d'une faillite des sécurités : l'un avait été exposé corps entier (il venait de se fiancer, avait des anomalies du caryotype, il a bien vécu et a pu avoir des enfants dix ans plus tard), un autre au niveau de la tête (sans suite non plus), d'autres ont pu être rassurés car l'enquête avait infirmé les premiers résultats, ou bien c'était le dosimètre qui était resté dans la blouse.

Quand la prévention technique a réussi à progresser, l'inquiétude a baissé, les doses aussi.

Ainsi depuis les années 1980, les doses lues sur les dosimètres passifs de l'IN2P3 sont toutes devenues minimes, les radioprotectionnistes étant finalement les plus exposés lors de tests mais à raison de moins de $1 \mathrm{mSv}$. La dose collective était très faible voire négligeable en Homme.Sievert. Nous avons alors limité le port des films passifs au strict nécessaire; des films trimestriels suffisaient éventuellement et nous avons cessé de les faire porter aux secrétaires ou aux personnels ayant des expositions potentielles rares, préférant les classer comme visiteurs occasionnels de la zone contrôlée. La grande rigueur dans la gestion, la lecture et le rendu des doses en temps utile a été très bénéfique aux recherches. 
Quant aux dosimètres de poignet et les dosimètres thermoluminescents (FLi) portés au doigt, ils ont servi chez les chirurgiens mais peu ou pas chez les chercheurs, si ce n'est parfois pour les rassurer.

Nous avons été gênés davantage, nous médecins du travail, par la faiblesse de la dosimétrie des neutrons auprès des accélérateurs de particules, surtout pour les neutrons de basse énergie, lorsque nous cherchions à corréler l'augmentation des cas de cancer éventuellement radio-induits aux longues années d'exposition aux neutrons.

L'avenir est encore à la prévention technique et l'évaluation de son efficacité par la dosimétrie.

\subsection{L'expérience du docteur Jean Luc Marande, médecin du travail de l'Assistance publique, hôpitaux de Paris (APHP)}

\subsubsection{Les expositions aux rayonnements ionisants dans les établissements de santé (ES)}

Trois types de rayonnements ionisants (RI) sont utilisés dans les établissements de santé : les photons $\mathrm{X}$ et $\gamma$ et les rayonnements $\beta$.

De nombreux acteurs de santé peuvent être exposés aux RI :

a) les médecins : les radiologues, les cardiologues, les gastro-entérologues, les hépatologues, les rhumatologues... les chirurgiens viscéraux, les orthopédistes, les urologues et les anesthésistes-réanimateurs pour les rayons $\mathrm{X}$ et les biophysiciens, les radiothérapeutes (et les neurochirurgiens) et les biologistes pour les rayonnements $\mathrm{X}, \beta$ et $\gamma$;

b) les dentistes et les stomatologistes pour les rayons $X$;

c) les paramédicaux : les manipulateurs d'électroradiologie médicale (MERM) et des personnels infirmiers et aides-soignants de certains secteurs pour les rayonnements $X, \beta$ et $\gamma$ et des techniciens de laboratoire et des agents des services hospitaliers travaillant dans des secteurs où sont manipulés des radioéléments $\beta$ et $\gamma$;

d) la personne compétente en radioprotection (PCR) pour les rayonnements $\mathrm{X}$, $\beta$ et $\gamma$;

e) le radio-physicien (PSRPM) lors des contrôles pour les rayonnements $X, \beta$ et $\gamma$

f) le radio-pharmacien lors des contrôles des médicaments en médecine nucléaire pour les radioéléments $\beta$ et $\gamma$; 
g) les agents du service biomédical pour les dépannages pour les rayonnements $\mathrm{X}, \beta$ et $\gamma$

h) les ouvriers des services techniques lors de travaux dans des secteurs utilisant des rayonnements $X, \beta$ et $\gamma$.

\subsubsection{Les sources scellées et appareils de radiologie}

Les personnels ayant un risque d'irradiation sont reportés dans le tableau I.

TABLEAU I

Personnels ayant un risque d'irradiation

\begin{tabular}{|c|c|c|c|c|c|c|c|}
\hline Secteur & médecin & dentiste & MERM & IDE & AS & $\mathrm{TL}$ & ASH \\
\hline Radiologie conventionnelle & radiologue & & + & & $(+)$ & & \\
\hline Radiologie exportée & & & + & $(+)$ & + & & + \\
\hline Tomodensitométrie & radiologue & & + & & + & & \\
\hline Mammographie & radiologue & & + & & & & \\
\hline Ostéodensitométrie & rhumatologue & & & & & & \\
\hline Stomatologie & & + & + & & $(+)$ & & \\
\hline $\begin{array}{l}\text { Actes radio-guidés invasifs } \\
\text { (interventionnel) }\end{array}$ & $\begin{array}{l}\text { radiologue cardiologue } \\
\text { hépatologue }\end{array}$ & & + & + & & & \\
\hline $\begin{array}{l}\text { Actes chirurgicaux avec un } \\
\text { amplificateur de brillance en } \\
\text { per-opératoire }\end{array}$ & $\begin{array}{l}\text { chir. viscéral orthopédiste } \\
\text { urologue anesthésistes- } \\
\text { réanimateurs }\end{array}$ & & $(+)$ & + & & & \\
\hline Endoscopie digestive & gastro-entérologue & & $(+)$ & + & & & \\
\hline Synoviorthèse $+\beta$ et $\gamma$ & rhumatologue & & + & + & + & + & \\
\hline Radiothérapie & radiothérapeute & & + & & $(+)$ & & \\
\hline Radio-chirurgie & $\begin{array}{l}\text { radiothérapeute neurochir- } \\
\text { urgien }\end{array}$ & & + & & $(+)$ & & \\
\hline Curiethérapie & radiothérapeute & & + & + & + & & + \\
\hline
\end{tabular}

MERM manipulateur en électroradiologie médicale ; IDE infirmier; AS aide-soignant; TL technicien de laboratoire ; ASH agent des services hospitaliers.

\subsubsection{Les sources non scellées}

Les personnels ayant un risque d'irradiation et/ou de contamination sont reportés dans le tableau II. 
TABLEAU II

Les personnels ayant un risque d'irradiation et/ou de contamination.

\begin{tabular}{|c|c|c|c|c|c|c|}
\hline secteur & médecin & MERM & IDE & $\overline{\text { AS }}$ & $\mathrm{TL}$ & ASH \\
\hline Médecine nucléaire in vivo & biophysicien & + & + & + & + & + \\
\hline Médecine nucléaire in vitro & $\begin{array}{l}\text { biophysicien } \\
\text { biologiste }\end{array}$ & & & + & + & + \\
\hline Curiethérapie métabolique & biophysicien & + & + & + & & + \\
\hline $\begin{array}{l}\text { Laboratoires de radio-analyse et de } \\
\text { recherche }\end{array}$ & biologiste & & & + & + & + \\
\hline
\end{tabular}

\subsubsection{Le suivi dosimétrique}

Chaque travailleur appelé à exécuter une opération en zone surveillée ou en zone contrôlée fait l'objet d'un suivi dosimétrique adapté au mode d'exposition. Les mesures de l'exposition externe ou interne sont réalisées soit par l'Institut de radioprotection et de sûreté nucléaire (IRSN), soit par un organisme ou un laboratoire d'analyses de biologie médicale titulaires d'un certificat d'accréditation et agréés par l'Autorité de sûreté nucléaire (ASN).

\subsubsection{La dosimétrie passive}

Le médecin du travail établit pour chaque travailleur un bilan dosimétrique afin de calculer la somme des doses efficaces reçues par exposition externe et interne et la somme des doses équivalentes.

\section{a) La dosimétrie passive thoracique, dite corps entier}

Elle est mensuelle ou trimestrielle. Elle est la mesure de référence utilisée pour le respect des valeurs limites. On utilise surtout deux types de dosimètre RPL (Radiophotoluminescence) ou OSL (Luminescence optiquement stimulée) dit InLight. Ils sont sensibles aux photons $\mathrm{X} / \gamma$ et aux rayons $\beta$ et peuvent être relus. Le seuil de détection est de 50 ou $100 \mu \mathrm{Sv}\left(10^{-6} \mathrm{~Sv}\right)$. Le dosimètre doit toujours être porté sous un éventuel vêtement radioprotecteur pendant le temps de travail exposant aux RI. Il mesure la dose efficace $E$. Elle est notée dans la feuille de résultats dosimétriques dans la colonne Profonde $\mathrm{Hp}(10)$.

\section{b) La dosimétrie passive par dosimètres thermoluminescents (TLD)}

Elle est utilisée chaque fois qu'il y a un risque démontré d'irradiation des extrémités (main, doigts) ou des yeux ou pour faire des études de poste. Le seuil de détection est de l'ordre de $300 \mu \mathrm{Sv}$. Les TLD sont sensibles, réutilisables et 
pratiques pour les mesures ponctuelles mais ils ne peuvent pas être relus. Ils sont surtout utilisés en bague pour connaître la dose peau au doigt de la main la plus exposée pendant les actes médicaux diagnostiques et thérapeutiques guidés par la radioscopie. Ils peuvent être également posés sur une protection latérale d'une paire de lunettes ou d'une visière de radioprotection pour calculer la dose à l'œil lors des actes exposants de la radiologie interventionnelle. Le TLD mesure une dose équivalente $\mathrm{H}$. Elle est notée dans la feuille de résultats dosimétriques dans la colonne Peau $\mathrm{Hp}(0,07)$.

\section{c) Les résultats}

Sous leur forme nominative, les résultats du suivi dosimétrique et les doses efficaces reçues sont communiqués au travailleur intéressé ainsi qu'au médecin désigné à cet effet par celui-ci et au médecin du travail dont il relève. Au vu de ces résultats, le médecin du travail peut prescrire, au titre de la surveillance médicale, les examens qu'il estime nécessaires. L'employeur peut avoir connaissance des résultats de la dosimétrie passive sous une forme excluant toute identification des travailleurs. Afin de procéder à l'évaluation prévisionnelle et à la définition des objectifs, avant la réalisation d'opérations dans la zone contrôlée ou surveillée, la personne compétente en radioprotection (PCR), demande communication des doses efficaces reçues sous une forme nominative sur une période de référence n'excédant pas les douze derniers mois. L'IRSN centralise l'ensemble des données dosimétriques et donne accès en ligne aux données des 12 mois glissants pour le médecin du travail, la PCR et le travailleur selon des règles précises grâce à son système d'information SISERI.

\subsubsection{Le suivi radiologique des personnels médicaux et non médicaux classés en catégorie A}

Selon le secteur d'activité, ce suivi comporte :

- dosimétrie passive thoracique mensuelle ;

- dosimétrie active ;

- dosimétrie des extrémités, des yeux (TLD) à la demande ;

- anthroporadiamétrie ou analyses radio-toxicologiques (urines) en cas de risque de contamination.

\subsubsection{Le suivi radiologique des personnels médicaux et non médicaux classés en catégorie B selon le secteur d'activité}

- dosimétrie passive thoracique trimestrielle ;

- dosimétrie active lors des opérations ponctuelles en zone contrôlée ; 
- anthroporadiamétrie ou analyses radiotoxicologiques des urines en cas de risque de contamination dans un secteur de médecine nucléaire ;

- dosimétrie des extrémités (TLD) couplée à des analyses radio-toxicologiques (urines) pour les techniciens de laboratoire exposés à des radionucléides $\beta$ de faible énergie.

\subsubsection{Réflexions du médecin du travail concernant la dosimétrie passive dans les ES}

\subsubsection{Suivi des femmes enceintes}

Les personnels étant très majoritairement jeunes et féminins, le souci permanent est la grossesse et l'allaitement pour les expositions aux sources non scellées. La réglementation précise que l'exposition de l'enfant à naître est, pendant le temps qui s'écoule entre la déclaration de grossesse et l'accouchement, aussi faible que raisonnablement possible, et en tout état de cause inférieur à 1 mSv (Art. D4152-5). Les femmes enceintes sont sensibilisées aux risques radiologiques pour elles et leurs enfants à naître et sont retirées précocement des secteurs justifiant d'un classement en catégorie A et de certains secteurs justifiant, certes d'un classement en catégorie $\mathrm{B}$, mais l'exposant à d'autres risques physiques, par exemple la radiologie exportée (radiographies au lit ou au bloc opératoire). L'exposition radiologique corps entier de ces femmes est faible voire très faible, l'exposition de leurs enfants à naître devient dès lors très faible. C'est malheureusement plus vrai pour les femmes du personnel paramédical que pour les femmes médecins qui travaillent dans les différents secteurs de radiologie interventionnelle (cardiologues, spécialités chirurgicales...). Afin de vérifier l'irradiation de l'enfant à naître, il est recommandé de faire porter à la femme un dosimètre électronique à la hauteur du bassin.

\subsubsection{Suivi des travailleurs exposés aux radionucléides $\beta$ de faible énergie}

Si le suivi dosimétrique corps entier est indispensable pour chaque travailleur exposé aux rayonnements pénétrants $\mathrm{X} / \gamma$, il a peu d'intérêt pour le suivi d'un travailleur uniquement exposé aux rayonnements peu pénétrants $\beta$. En revanche, un suivi dosimétrique des extrémités est très pertinent. À ce jour, la réglementation ne permet pas un suivi des extrémités sans suivi de l'organisme entier. Il faudrait revoir ce point réglementaire.

\subsubsection{Suivi des médecins}

Pendant des décennies, le suivi des médecins exposés aux RI consistait en un simple suivi dosimétrique. Depuis quelques années, ce suivi est couplé à une 
surveillance médicale renforcée. Ces visites permettent de sensibiliser les médecins au port des dosimètres passifs en particulier dans les blocs opératoires.

$\mathrm{Au}$ total, de nombreuses personnes exerçant des métiers médicaux et paramédicaux différents sont exposées aux RI dans les ES. Le suivi du risque radiologique est variable selon que le travailleur a un risque d'exposition externe ou interne ou externe et interne de manière intermittente ou permanente pendant son temps de travail. Le suivi des femmes enceintes est une priorité dans ces établissements. 